\title{
Parameterized Approximation of Dominating Set Problems
}

\author{
Rodney G. Downey ${ }^{1}$, Michael R. Fellows ${ }^{2}$, Catherine McCartin ${ }^{3}$, and Frances \\ Rosamond $^{2}$ \\ 1 Victoria University, Wellington, New Zealand, \\ Rod.Downey@mcs . vuw . ac.nz \\ 2 The University of Newcastle, Callaghan, Australia, \\ \{michael.fellows, frances.rosamond\}@newcastle.edu.au \\ 3 Massey University, Palmerston North, New Zealand, \\ C.M.McCartin@massey.ac.nz
}

\begin{abstract}
A problem open for many years is whether there is an FPT algorithm that given a graph $G$ and parameter $k$, either: (1) determines that $G$ has no $k$-Dominating SET, or (2) produces a dominating set of size at most $g(k)$, where $g(k)$ is some fixed function of $k$. Such an outcome is termed an FPT approximation algorithm. We describe some results that begin to provide some answers. We show that there is no such FPT algorithm for $g(k)$ of the form $k+c$ (where $c$ is a fixed constant, termed an additive FPT approximation), unless FPT $=W[2]$. We answer the analogous problem completely for the related INDEPENDENT Dominating SET (IDS) problem, showing that IDS does not admit an FPT approximation algorithm, for any $g(k)$, unless $F P T=W[2]$.
\end{abstract}

\section{Introduction}

Most of the work in the area of parameterized complexity and algorithmics to date has focused on exact algorithms for decision problems. However, the theory of parameterized complexity, which derives from the contrast between timecost functions of the form $f(k) n^{c}$ (fixed-parameter tractability, FPT, ) and those of the form $n^{g(k)}$, where $n$ is the overall input size, and $k$ is a relevant secondary parameter of the situation, can clearly be deployed in a variety of ways in analyzing computational complexity. In particular, the ideas of such multivariate complexity analysis can be applied to counting problems [7,11], online problems $[11,6]$, in analyzing approximation complexity (PTASs versus EPTASs) [9] and in exploring $k$-speedups of local search heuristics [10].

We report here on some concrete results concerning a formulation of approximation complexity that arises specifically and naturally in the context of parameterized problems and the central notion of fixed-parameter tractability. Consider the following parameterized computational problem specification.

Dominating Set $g(k)$-Approximation

Input: A graph $G$ and a positive integer $k$. 
Parameter: $k$

Output: Either: (1) A determination that $G$ does not admit a dominating set of size at most $k$, or (2) a dominating set for $G$ of size at most $g(k)$.

Can this problem be solved in FPT time?

The subject of parameterized complexity is by now so well known that we assume some basic familiarity with the main ideas, such as presented in $[5,7$, 13]. The concrete question above concerning parameterized approximation of the Dominating SET problem has been open for many years, and is still unresolved. What is well-known is that determining whether a graph $G$ has a dominating set of size $k$ (the exact formulation of the problem) is complete for the parameterized complexity class $W[2]$.

The problem specification defined above that asks for an FPT $g(k)$-approximation for a parametrically intractable problem (i.e., Dominating SET) is clearly just one example of a general kind of approximation issue that one can pose for any parametrically intractable optimization problem.

At the present time, for concrete problems, very little is known about the subject of FPT approximability for $W$-hard problems. The general subject of the interactions of parameterized complexity and approximation is well-surveyed in [9]. See also the recent papers [3,2]. Magdelena Grüber and Martin Grohe have recently shown that the $W[1]$-hard problem of determining whether a directed graph has $k$ vertex disjoint directed cycles has an FPT approximation [8].

Here we report on two concrete results concerning the parameterized approximability of graph domination problems. We show that:

(1) The Dominating Set problem has no $g(k)$-FPT approximation algorithm for $g(k)=k+c($ for any constant $c)$ unless $F P T=W[2]$.

(2) The IndePendent Dominating SET problem has no $g(k)$-FPT approximation algorithm, for any $g(k)$, unless $F P T=W[2]$.

\section{No additive parameterized approximation for Dominating Set}

In this section we consider the question of whether the Dominating SET problem has an additve FPT approximation algorithm, meaning a $g(k)$-FPT approximation where $g(k)=k+c$ for a constant $c$.

Theorem 1. Add-Approx $k$-Dominating Set is $W[2]$-hard.

Proof: We transform from $k$-Dominating SET.

Let $G=(V, E)$ be a graph and let $k$ be the parameter. We produce $G^{\prime}=\left(V^{\prime}, E^{\prime}\right)$ such that $G^{\prime}$ has a $c$-additive approximate solution for $d k$-DominATing SET, that is, $G^{\prime}$ contains a dominating set of size at most $d k+c$ if and only if $G$ contains a dominating set of size at most $k$.

Consider the graph $G^{\prime}$ that simply consists of $c+1$ copies of $G$. If $G$ has a $k$ dominating set, then clearly $G^{\prime}$ has a dominating set of size at most $k^{\prime}=(c+1) k$. 
If $G^{\prime}$ has a dominating set that approximates $k^{\prime}$ within the additive constant $c$, that is, if $G^{\prime}$ has a dominating set of size at most $k^{\prime}+c$, then one of the $c+1$ copies witnesses the fact that $G$ has a $k$-dominating set.

\section{A completely inapproximable parameterized domination problem}

In this section we show that $k$-IndePendent Dominating SET is completely inapproximable. The problem is formally defined:

$g(k)$-Approximate Independent Dominating Set

Input: $G=(V, E)$

Parameter: $k$

Output: 'NO' asserting that no independent dominating set $V^{\prime} \subseteq V$ of size $\leq k$ for $G$ exists, or an independent dominating set $V^{\prime} \subseteq V$ for $G$ of size at most $g(k)$.

Theorem 2. There is no FPT algorithm for $k$-APPROXIMATE INDEPENDENT Dominating SET for any computable function $g(k)$ unless $W[2]=F P T$.

Proof: The proof is a reduction from the $W[2]$-complete Dominating SeT problem, parameterized by the solution size. Let $G=(V, E)$ be a graph for which we wish to determine if it has a dominating set of size at most $k$. We use $[t]$ to denote the set $\{1, \ldots, t\}$. We construct a graph $G^{\prime}$ as follows. The vertex set of $G^{\prime}$ consists of the following sets.

$S=\{s[r, i]: 1 \leq r \leq k, i \in[g(k)+1]\} \quad$ The sentinel vertices.

$C=\{c[r, u]: 1 \leq r \leq k, u \in V\} \quad$ The choice vertices.

$T=\{t[u, i]: u \in V, i \in[g(k)+1]\} \quad$ The test vertices.

The edge set $E^{\prime}$ of $G^{\prime}$ consists of the following sets of edges.

$$
\begin{aligned}
& E^{\prime}(1)=\{s[r, i] c[r, u]: 1 \leq r \leq k, i \in[g(k)+1], u \in V\} \\
& E^{\prime}(2)=\left\{c[r, u] c\left[r, u^{\prime}\right]: 1 \leq r \leq k, u, u^{\prime} \in V\right\} \\
& E^{\prime}(3)=\left\{c[r, u] t[v, i]: 1 \leq r \leq k, u \in V, v \in N_{G}[u], i \in[g(k)+1]\right\}
\end{aligned}
$$

Central to the construction are $k$ groups of choice vertices, each forming a clique, by the edges of $E^{\prime}(2)$. Corresponding to each of these $k$ cliques is a set of $g(k)+1$ sentinel vertices, and the edges of $E^{\prime}(1)$ connect each sentinel to all of the vertices in its corresponding choice clique. The sentinel vertices form an independent set in $G^{\prime}$, as do the test vertices. The edges of $E^{\prime}(3)$ connect the choice vertices to the test vertices in the natural way, reflecting the structure of G. 


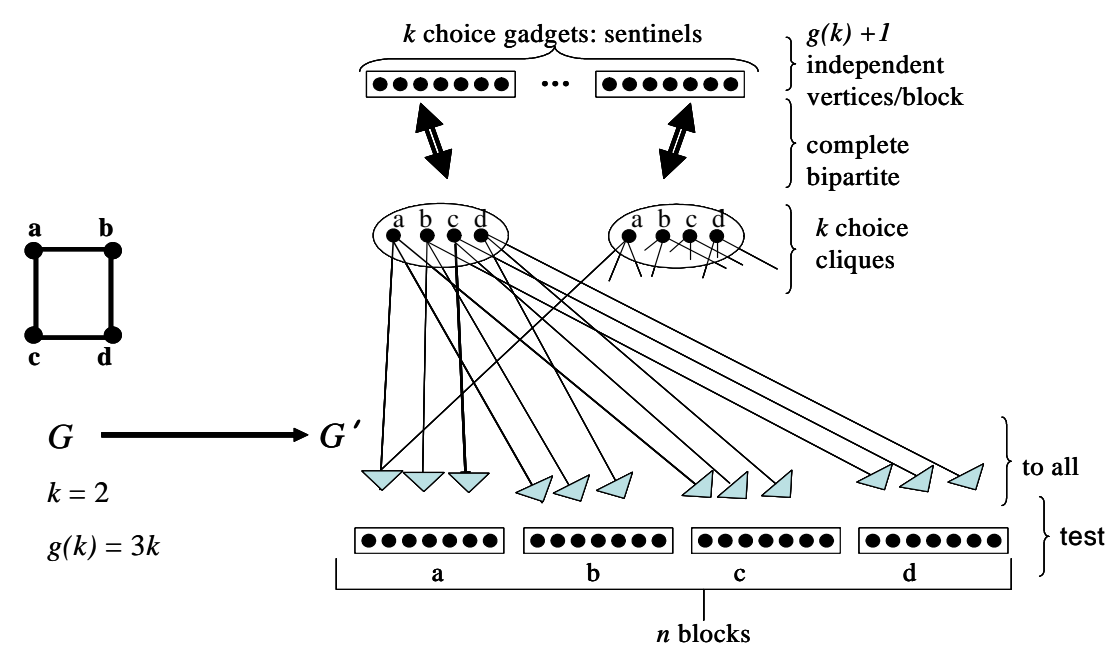

Fig. 1. An example of the reduction.

An example of the construction is shown in Figure 1.

We argue the correctness of the reduction. Establishing the following two claims is sufficient.

Claim 1. If $G$ has a $k$-dominating set then $G^{\prime}$ has an independent dominating set of size $k$.

Proof of Claim 1. Let $v_{1}, \ldots, v_{k}$ be a dominating set in $G$ of size $k$. It is easy to check that the corresponding vertices of $G^{\prime}$, one in each of the choice vertex cliques of $G^{\prime}$, forms an independent $k$-dominating set in $G^{\prime}$.

Claim 2. If $G^{\prime}$ has an independent dominating set of size at most $g(k)$, then $G$ has a dominating set of size at most $k$.

Proof of Claim 2. Suppose $G^{\prime}$ has a dominating set $D^{\prime}$ of size at most $g(k)$. There must be at least one vertex $c\left[i, u_{i}\right] \in D^{\prime}$ in each of the $k$ choice vertex cliques, $1 \leq i \leq k$, for the reason that otherwise the sentinels could not be dominated. Since $D^{\prime}$ is an independent set, we can conclude that there is exactly one vertex of $D^{\prime}$ in each of the $k$ choice cliques. Let $D$ be set of at most $k$ vertices of $G$ that these choice vertices of $D^{\prime}$ indicate, $D=\left\{u_{i}: 1 \leq i \leq k\right\}$. (Note that while there are $k$ choice cliques, a vertex could be thus indicated more than once, so we only know that $|D| \leq k$.) We argue that $D$ is a dominating set in $G$. By the construction of $G^{\prime}$, this follows from the fact that for all $v \in V$, there is at least one test vertex $t[v, i]$ corresponding to $v$ in $G^{\prime}$ that does not belong to $D^{\prime}$, and must be dominated by a vertex in $C$. The definition of the set of edges $E^{\prime}(3)$ of $G^{\prime}$ allows us to conclude that $D$ is a dominating set in $G$. 


\section{Summary and Open Problems}

We have described two results exploring the parameterized approximability of Dominating Set and Independent Dominating Set. So far as we are aware, our result on IDS is the first result showing that a natural parameterized problem does not admit any parameterized approximation unless the W-Hierarchy collapses. Many questions in this new area of investigation remain open.

(1) We have shown that there is no additive parameterized approximation for Dominating Set. Can this be amplified somehow to show that DS does not admit a multiplicative parameterized approximation, unless the W-Hierarchy collapses?

(2) There are many variants of the Dominating SET problem. What can be shown about the parameterized approximability or inapproximability of these problems?

(3) As a technical issue, might the PCP Theorem (or some parameterized analog) prove useful in exploring questions concerning parameterized approximability?

(4) If one attempts to adapt our reduction for IDS to DS, great differences between the two problems quickly become apparent. It is conceivable that DS does admit an FPT parameterized approximation. Can this perhaps be shown for restricted classes of graphs? For example, it has recently been shown that DS remains $W[2]$-complete for graphs of diameter 2 [12], so the question remains interesting for this seemingly quite restricted class.

\section{References}

1. C. Bazgan: Schemas d'Approximation et Complexite Parametree. Rapport de DEA, Universite Paris Sud, 1995.

2. Y. Chen, M. Grohe and M Grüber: On Parameterized Approximability. In H.L. Bodlaender and M.A. Langston, editors, Proceedings of the Second International Workshop on Parameterized and Exact Computation, Springer-Verlag, Lecture Notes in Computer Science 4169 (2006), 96-108.

3. L. Cai, X. Huang: Fixed Parameter Approximation: Conceptual Framework and Approximability Results. In H.L. Bodlaender and M.A. Langston, editors, Proceedings of the Second International Workshop on Parameterized and Exact Computation, Springer-Verlag, Lecture Notes in Computer Science 4169 (2006), 96-108.

4. M. Cesati, L. Trevisan: On the Efficiency of Polynomial Approximation Schemes. Information Processing Letters, 64(4), pp 165-171, 1997.

5. R. G. Downey, M. R. Fellows: Parameterized Complexity Springer-Verlag, 1999.

6. R. G. Downey, C. M. McCartin: Online Problems, Pathwidth, and Persistence. Proceedings of IWPEC 2004, Springer-Verlag LNCS 3162, pp 13-24, 2004.

7. J. Flum, M. Grohe: The Parameterized Complexity of Counting Problems. SIAM Journal on Computing, 33(4), pp 892-922, 2004.

8. M. Grohe and M. Grüber: Parameterized Approximability of the Disjoint Cycle Problem. Proceedings of ICALP 2007, Springer-Verlag, Lecture Notes in Computer Science 4596, pp 363-374, 2007. 
9. D. Marx: Parameterized Complexity and Approximation Algorithms. The Computer Journal, 51(1), pp 60-78, 2008.

10. D. Marx: Searching the k-Change Neighborhood for TSP is W[1]-hard. Operations Resarch Letters, 36(1), pp 31-36, 2008.

11. C. M. McCartin: Contributions to Parameterized Complexity, Ph.D. Thesis, Victoria University, Wellington, 2003.

12. C. M. McCartin, P. Rossmanith and M. Fellows: Frontiers of Intractability for Dominating Set, Manuscript, 2007.

13. R. Niedermeier: Invitation to Fixed-Parameter Algorithms. Oxford University Press, 2006. 